\title{
MDS 2D convolutional codes with optimal 1D horizontal projections
}

\author{
Paulo Almeida · Diego Napp · Raquel \\ Pinto
}

Received: date / Accepted: date

\begin{abstract}
Two dimensional (2D) convolutional codes is a class of codes that generalizes standard one-dimensional (1D) convolutional codes in order to treat two dimensional data. In this paper we present a novel and concrete construction of $2 \mathrm{D}$ convolutional codes with the particular property that their projection onto the horizontal lines yield optimal (in the sense of [2]) 1D convolutional codes with a certain rate and certain Forney indices. Moreover, using this property we show that the proposed constructions are indeed Maximum Distance Separable (MDS), i.e., are 2D convolutional codes having the maximum possible distance among all $2 \mathrm{D}$ convolutional codes with the same parameters. The key idea is to use a particular type of superregular matrices to build the generator matrix.
\end{abstract}

Keywords 2D convolutional codes - optimal codes $\cdot$ MDS codes $\cdot$ superregular matrices

Mathematics Subject Classification (2000) 94B10 15 B33 11 T71

\section{Introduction}

One of the fundamental tasks of coding theory is the construction of codes having as large distance as possible. In the context of block codes this task has been highly developed and there exist numerous sophisticated classes of such codes, e.g., Reed-Solomon, BCH, Golay, etc. In contrast to block codes,

Paulo Almeida · Diego Napp · Raquel Pinto

CIDMA - Center for Research and Development in Mathematics and Applications, Department of Mathematics, University of Aveiro, Aveiro, Portugal.

E-mail: palmeida@ua.pt,diego@ua.pt and raquel@ua.pt

This work was supported by Portuguese funds through the CIDMA - Center for Research and Development in Mathematics and Applications, and the Portuguese Foundation for Science and Technology (FCT-Fundação para a Ciência e a Tecnologia), within project UID/MAT/04106/2013. 
the construction of good convolutional codes has not been fully understood. In fact, many (one-dimensional - 1D) convolutional codes use in practice have been found by computer searches and their distance properties must be also computed by full search. In recent years a great deal of effort has been dedicated to develop constructions of nonbinary convolutional codes having good distance $[4,8,13,18-20,22,25]$. It has been shown that by taking advantage of their rich mathematical structure, convolutional codes can have indeed very large distances and correct many erasures patterns that block codes fail to correct $[6,28]$.

Tied up to the construction of codes with large distance, and in particular of Maximum Distance Separable (MDS), is the notion of superregular matrices. It is well known that a block code with encoder matrix $G=[I A]^{T}$ is MDS if and only if $A$ is full superregular, i.e., all minors of $A$ are nonzero. In the context of convolutional codes it has been shown in [8] that (strongly) MDS (and also Maximum Distance Profile) are closely related to the notion of lower triangular (LT) superregular matrices, i.e., lower triangular Toeplitz matrices having all their nontrivial minors (to be formally defined later) nonzero.

A second stage in the theory of convolutional codes resulted on the development of the theory of multidimensional convolutional codes $(n \mathrm{D})[7,14,21$, 29 . Roughly speaking $1 \mathrm{D}$ convolutional codes can be seen as a generalization of block codes in the sense that a block code is a convolutional code with no delay, i.e., block codes are basically 0D convolutional codes. In this way, multidimensional $(n \mathrm{D})$ convolutional codes extend the notion of block codes and $1 \mathrm{D}$ convolutional codes. During the last few decades there has been a renewed interest in convolutional codes for multidimensional data [3,12] motivated mainly by the possibility of encoding data recorded in $n$ dimensions, e.g., pictures, videos, storage media, etc. Moreover, $n \mathrm{D}$ convolutional codes have been a fruitful source of problems and conjectures both in signal processing [3] and algebra of $n$-variate polynomial matrices [14].

Although the algebraic properties of those codes have been already studied [30] very little is known about their distance properties. The main goal of this paper is to study constructions of two-dimensional (2D) convolutional codes having large distance. The idea used here is to build 2D convolutional codes in such a way that when we project them into certain 1D lines we obtain optimal 1D convolutional codes. Summing up the distances of these optimal 1D codes, we show that the proposed 2D convolutional code is MDS. This idea is similar to the one used in [17] where the projection is done (only) onto the axes. In this paper we refine this idea and project onto horizontal lines which allows us to show that the proposed codes achieve the generalized 2D Singleton bound [5], i.e., they are MDS 2D convolutional codes. The constructions presented here used very different techniques than the ones used in [5] where Cauchy circulant matrices were used. Moreover, the constructions presented in [5] were restricted to certain set of parameters. In this paper we rather make use of a 
very general class of superregular matrices recently introduced in $[1,2]$. These matrices completely generalize previous notions of superregular matrices and therefore allow to be applied to the involved class of $2 \mathrm{D}$ convolutional codes.

The paper is organized as follows: In section 2 we recall the necessary background of both 1D and 2D finite support convolutional codes. Section 3 is devoted to the notion of superregular matrices where several properties related to these matrices are proved or recalled. Finally in section 4 we present a novel class of 2D convolutional codes built upon the introduced type of superregular matrices and study their properties.

\section{Convolutional codes}

2.1 One-dimensional (1D) convolutional codes

Let $\mathbb{F}$ be a finite field and $\mathbb{F}[z]$ the ring of polynomials with coefficients in $\mathbb{F}$. A (finite support) convolutional code $\mathcal{C}$ of rate $k / n$ is an $\mathbb{F}[z]$-submodule of $\mathbb{F}[z]^{n}$, where $k$ is the rank of $\mathcal{C}$ (see $[16,23]$ ). The elements of $\mathcal{C}$ are called codewords.

A full column rank matrix $G(z) \in \mathbb{F}[z]^{n \times k}$ whose columns constitute a basis for $\mathcal{C}$ is called an encoder of $\mathcal{C}$. So,

$$
\begin{aligned}
\mathcal{C} & =\operatorname{Im}_{\mathbb{F}[z]} G(z) \\
& =\left\{v(z) \in \mathbb{F}[z]^{n}: v(z)=G(z) u(z) \text { with } u(z) \in \mathbb{F}[z]^{k}\right\} .
\end{aligned}
$$

Two full column rank matrices $G_{1}(z), G_{2}(z) \in \mathbb{F}[z]^{n \times k}$ are said to be equivalent encoders if $\operatorname{Im}_{\mathbb{F}[z]} G_{1}(z)=\operatorname{Im}_{\mathbb{F}[z]} G_{2}(z)$, which happens if and only if there exists a unimodular (square matrix with determinant in $\mathbb{F} \backslash\{0\}$ ) matrix $U(z) \in \mathbb{F}[z]^{k \times k}$ (see [23]) such that $G_{2}(z)=G_{1}(z) U(z)$.

Among the encoders of the code, the column reduced are the ones with smallest sum of the column degrees. Moreover, two equivalent column reduced encoders have the same column degrees up to a permutation. Such degrees are called the Forney indices of the code. The number of Forney indices with a certain value $\nu$ is called the multiplicity of $\nu$. The degree (or complexity) of a convolutional code is the sum of the Forney indices of the code.

The weight of a word $v(z)=\sum_{i \geq 0} v_{i} z^{i} \in \mathbb{F}[z]^{n}$ is given by

$$
\mathrm{wt}(v(z))=\sum_{i \in \mathbb{N}} \mathrm{wt}\left(v_{i}\right),
$$

where the weight of a constant vector $v_{i} \in \mathbb{F}^{n}$ is the number of nonzero entries of $v_{i}$ and the distance of a nontrivial 1D convolutional code $\mathcal{C}$ is defined as

$$
\operatorname{dist}(\mathcal{C})=\min \{\operatorname{wt}(v(z)): v(z) \in \mathcal{C}, \text { with } v(z) \neq 0\} .
$$

The following theorem gives an upper bound on the free distance of a convolutional code. 
Theorem 1 [25, theorem 2.1] Let $\mathcal{C}$ be a convolutional code with rate $k / n$ and distinct Forney indices $\nu_{1}<\cdots<\nu_{r}$ with corresponding multiplicities $m_{1}, \ldots, m_{r}$. Then the distance of $\mathcal{C}$ must satisfy

$$
\operatorname{dist}(\mathcal{C}) \leq n\left(\nu_{1}+1\right)-m_{1}+1
$$

A convolutional code of rate $k / n$ with different Forney indices $\nu_{1}<\cdots<\nu_{r}$ with corresponding multiplicities $m_{1}, \ldots, m_{r}$ and distance $n\left(\nu_{1}+1\right)-m_{1}+1$ is said to be an optimal $\left(n, k, \nu_{1}, m_{1}\right)$ convolutional code.

\subsection{Two-dimensional (2D) convolutional codes}

Two-dimensional (2D) convolutional codes naturally extend the notion of 1D convolutional codes, see for instance $[5,14,22,30]$. We are going to consider convolutional codes whose codewords belong to $\mathbb{F}\left[z_{1}, z_{2}\right]^{n}$, where $\mathbb{F}\left[z_{1}, z_{2}\right]$ is the ring of polynomials in two unknowns with coefficients in $\mathbb{F}$. Such codes are called 2D (finite support) convolutional codes. More precisely, a 2D (finite support) convolutional code $\mathcal{C}$ of rate $k / n$ is a free $\mathbb{F}\left[z_{1}, z_{2}\right]$-submodule of $\mathbb{F}\left[z_{1}, z_{2}\right]^{n}$ of rank $k$ (see $\left.[7,30]\right)$. An encoder of $\mathcal{C}$ is a full column rank matrix $G\left(z_{1}, z_{2}\right) \in \mathbb{F}\left[z_{1}, z_{2}\right]^{n \times k}$ whose columns constitute a basis for $\mathcal{C}$. Therefore,

$$
\begin{aligned}
\mathcal{C} & =\operatorname{Im}_{\mathbb{F}\left[z_{1}, z_{2}\right]} G\left(z_{1}, z_{2}\right) \\
& =\left\{v\left(z_{1}, z_{2}\right) \in \mathbb{F}\left[z_{1}, z_{2}\right]^{n}: v\left(z_{1}, z_{2}\right)=G\left(z_{1}, z_{2}\right) u\left(z_{1}, z_{2}\right), u\left(z_{1}, z_{2}\right) \in \mathbb{F}\left[z_{1}, z_{2}\right]^{k}\right\} .
\end{aligned}
$$

Two encoders $G_{1}\left(z_{1}, z_{2}\right), G_{2}\left(z_{1}, z_{2}\right) \in \mathbb{F}\left[z_{1}, z_{2}\right]^{n \times k}$ are said to be equivalent if

$$
\operatorname{Im}_{\mathbb{F}\left[z_{1}, z_{2}\right]} G_{1}\left(z_{1}, z_{2}\right)=\operatorname{Im}_{\mathbb{F}\left[z_{1}, z_{2}\right]} G_{2}\left(z_{1}, z_{2}\right),
$$

which happens if and only if $G_{1}\left(z_{1}, z_{2}\right)=G_{2}\left(z_{1}, z_{2}\right) U\left(z_{1}, z_{2}\right)$ for some unimodular matrix $U\left(z_{1}, z_{2}\right) \in \mathbb{F}\left[z_{1}, z_{2}\right]^{k \times k}$ (see $[29,30]$ ).

The weight of a word

$$
v\left(z_{1}, z_{2}\right)=\sum_{(i, j) \in \mathbb{N}^{2}} v_{i, j} z_{1}^{i} z_{2}^{j} \in \mathbb{F}^{n}\left[z_{1}, z_{2}\right]
$$

is defined in a similar way to the $1 \mathrm{D}$ case as

$$
\operatorname{wt}\left(v\left(z_{1}, z_{2}\right)\right)=\sum_{(i, j) \in \mathbb{N}^{2}} \operatorname{wt}\left(v_{i, j}\right),
$$

and the distance of $\mathcal{C}$ as

$$
\operatorname{dist}(\mathcal{C})=\min \left\{\operatorname{wt}\left(v\left(z_{1}, z_{2}\right)\right): v\left(z_{1}, z_{2}\right) \in \mathcal{C}, \text { with } v\left(z_{1}, z_{2}\right) \neq 0\right\} .
$$

We define the degree of a polynomial in two unknowns

$$
p\left(z_{1}, z_{2}\right)=\sum_{(i, j) \in \mathbb{N}^{2}} p_{i, j} z_{1}^{i} z_{2}^{j}
$$


as $\max \left\{i+j: p_{i, j} \neq 0\right\}$. Let $\mu_{i}$ be the column degree of the $i$-th column of a polynomial matrix $G\left(z_{1}, z_{2}\right)$, i.e, the maximum degree of the entries of the $i$-th column of $G\left(z_{1}, z_{2}\right)$. The external degree of $G\left(z_{1}, z_{2}\right)$ is the sum of its column degrees, i.e., $\sum_{i=1}^{k} \mu_{i}$. The degree of $\mathcal{C}$ is defined as the minimum of the external degrees among all the encoders of $\mathcal{C}[5]$.

If $\mathcal{C}$ is a $2 \mathrm{D}$ convolutional code of rate $k / n$ and degree $\delta$, then (see $[4,5])$

$$
\operatorname{dist}(\mathcal{C}) \leq \frac{\left(\left\lfloor\frac{\delta}{k}\right\rfloor+1\right)\left(\left\lfloor\frac{\delta}{k}\right\rfloor+2\right)}{2} n-k\left(\left\lfloor\frac{\delta}{k}\right\rfloor+1\right)+\delta+1 .
$$

Such bound is called the $2 \mathbf{D}$ generalized Singleton bound and if the distance of $\mathcal{C}$ equals such a bound, $\mathcal{C}$ is said to be Maximum Distance Separable (MDS). If $\mathcal{C}$ is a $2 \mathrm{D}$ convolutional code of rate $k / n$ and degree $\delta$ and $G\left(z_{1}, z_{2}\right)$ is an encoder of $\mathcal{C}$ with minimal external degree, then $G\left(z_{1}, z_{2}\right)$ must have $t=k\left(\left\lfloor\frac{\delta}{k}\right\rfloor+1\right)-\delta$ columns of degree $\left\lfloor\frac{\delta}{k}\right\rfloor$ and $k-t=\delta-k\left\lfloor\frac{\delta}{k}\right\rfloor$ columns of degree $\left\lfloor\frac{\delta}{k}\right\rfloor+1$. In the systems literature, the above set of indices are referred to as the generic set of column indices, [16, Corollary 4.3], see also [25, pag. 2046].

In [5] it was proved that there exist MDS 2D convolutional codes of rate $k / n$ and degree $\delta$ for

$$
n \geq k \frac{\left(\left\lfloor\frac{\delta}{k}\right\rfloor+2\right)\left(\left\lfloor\frac{\delta}{k}\right\rfloor+3\right)}{2}, \text { if } k \nmid \delta
$$

and

$$
n \geq k \frac{\left(\left\lfloor\frac{\delta}{k}\right\rfloor+1\right)\left(\left\lfloor\frac{\delta}{k}\right\rfloor+2\right)}{2}, \text { if } k \mid \delta,
$$

by providing constructions of such codes by means of Cauchy circulant matrices. However, the existence of such codes is not known for smaller $n$. In this paper, we give constructions of MDS $2 \mathrm{D}$ convolutional codes of rate $k / n$ and degree $\delta$, for

$$
n \geq k\left(\left\lfloor\frac{\delta}{k}\right\rfloor+1\right) .
$$

For that, in the next section, we use a type of superregular matrices similar to the ones used in $[1,8,15]$.

\section{Superregular matrices}

In this section, we recall the necessary definitions on superregular matrices and introduce a particular class of superregular matrices that we will use to build 2D convolutional codes. Such matrices have some similarities with the ones introduced in $[8,11]$. They have similar entries and, therefore, some properties are the same, even if the structure of these new matrices is different. 
Let $A=\left[\mu_{i \ell}\right]$ be a square matrix of order $m$ over $\mathbb{F}$ and $S_{m}$ the symmetric group of order $m$. The determinant of $A$ is given by

$$
|A|=\sum_{\sigma \in S_{m}} \operatorname{sgn}(\sigma) \mu_{1 \sigma(1)} \cdots \mu_{m \sigma(m)} .
$$

A trivial term of the determinant is a term $\mu_{\sigma}=\mu_{1 \sigma(1)} \cdots \mu_{m \sigma(m)}$, with at least one component $\mu_{i \sigma(i)}$ equal to zero. If $A$ is a square submatrix of a matrix $B$ with entries in $\mathbb{F}$, and all the terms of the determinant of $A$ are trivial, we say that $|A|$ is a trivial minor of $B$ (if $B=A$ we simply say that $|A|$ is a trivial minor). We say that a matrix $B$ is superregular if all its nontrivial minors are different from zero.

We note that there exist several notions of superregular matrices in the literature (see for instance $[8-11,24,26,27]$ ). These definitions depend on the type of blocks of zeros they have, if any. The definition given above generalizes all those notions.

The next results were derived in [2] and they will be very useful for our purposes in the next section. We start with a matrix theoretic theorem that establishes an optimality result on the number of nonzero entries of a vector in the right-image of a superregular matrix.

Theorem 2 [2, Theorem 3.1] Let $\mathbb{F}$ be a field and $a, b \in \mathbb{N}$, such that $a \geq b$ and $B \in \mathbb{F}^{a \times b}$. Suppose that $u=\left[u_{i}\right] \in \mathbb{F}^{b \times 1}$ is a column matrix such that $u_{i} \neq 0$ for all $1 \leq i \leq b$. If $B$ is a superregular matrix and every row of $B$ has at least one nonzero entry then $\operatorname{wt}(B u) \geq a-b+1$.

The next theorem (theorem 3.2 of [2]) gives sufficient conditions in order for a matrix to be superregular. A similar idea was developed in theorem 3.2 of [1], the novelty here is condition 2., which informally says that if we have a zero in a certain entry then all the entries Below or all the entries Before are also zero.

Theorem 3 (BB) [2, Theorem 3.3] Let $\alpha$ be a primitive element of a finite field $\mathbb{F}=\mathbb{F}_{p^{N}}$ and $B=\left[\nu_{i \ell}\right]$ be a matrix over $\mathbb{F}$ with the following properties

1. if $\nu_{i \ell} \neq 0$ then $\nu_{i \ell}=\alpha^{\beta_{i \ell}}$ for $\beta_{i \ell} \in\left\{1, \ldots, p^{N}-2\right\}$;

2. If $\nu_{i \ell}=0$ then $\nu_{i^{\prime} \ell}=0$, for any $i^{\prime}>i$ or $\nu_{i \ell^{\prime}}=0$, for any $\ell^{\prime}<\ell$;

3. if $\ell<\ell^{\prime}, \nu_{i \ell} \neq 0$ and $\nu_{i \ell^{\prime}} \neq 0$ then $2 \beta_{i \ell} \leq \beta_{i \ell^{\prime}}$;

4. if $i<i^{\prime}, \nu_{i \ell} \neq 0$ and $\nu_{i^{\prime} \ell} \neq 0$ then $2 \beta_{i \ell} \leq \beta_{i^{\prime} \ell}$.

Suppose $N$ is greater than any exponent of $\alpha$, that may appear in a nontrivial term of any minor of $B$. Then $B$ is superregular.

Some of the matrices that we are going to consider later satisfy all of the above conditions, but others will have, instead of 2., the following condition, which states that if we have a zero in a certain entry then all the entries After or all the entries Above are also zero.

$2^{\prime}$ If $\nu_{i \ell}=0$ then $\nu_{i^{\prime} \ell}=0$, for any $i^{\prime}<i$ or $\nu_{i \ell^{\prime}}=0$, for any $\ell^{\prime}>\ell$. 
The result is still valid and the proof follows the same reasoning employed in the proof of theorem 3.2 in [2], or can be obtained after a transposition of the matrix.

Theorem 4 (AA) Let $\alpha$ be a primitive element of a finite field $\mathbb{F}=\mathbb{F}_{p^{N}}$ and $B=\left[\nu_{i \ell}\right]$ be a matrix over $\mathbb{F}$ with the following properties

1. if $\nu_{i \ell} \neq 0$ then $\nu_{i \ell}=\alpha^{\beta_{i \ell}}$ for $\beta_{i \ell} \in\left\{1, \ldots, p^{N}-2\right\}$;

2. If $\nu_{i \ell}=0$ then $\nu_{i^{\prime} \ell}=0$, for any $i^{\prime}<i$ or $\nu_{i \ell^{\prime}}=0$, for any $\ell^{\prime}>\ell$;

3. if $\ell<\ell^{\prime}, \nu_{i \ell} \neq 0$ and $\nu_{i \ell^{\prime}} \neq 0$ then $2 \beta_{i \ell} \leq \beta_{i \ell^{\prime}}$;

4. if $i<i^{\prime}, \nu_{i \ell} \neq 0$ and $\nu_{i^{\prime} \ell} \neq 0$ then $2 \beta_{i \ell} \leq \beta_{i^{\prime} \ell}$.

Suppose $N$ is greater than any exponent of $\alpha$, that may appear in a nontrivial term of any minor of $B$. Then $B$ is superregular.

Proof If the matrix $B$ satisfies the assumptions of the theorem then $B^{T}$ satisfies the assumptions of theorem BB. Therefore, $B^{T}$ is superregular. By the definition of superregularity, $B$ is also superregular.

In [25, pag. 2047] it was left as an open problem the existence of convolutional codes having Forney indices $\nu_{1}<\cdots<\nu_{\ell}$ and free distance equal to the upperbound (1). In other words, the existence of optimal convolutional codes for any given set of Forney indices was left as an open question. In [2], it was considered a special class of matrices that allowed to exhibit convolutional codes with this property. These constructions will be very useful for our construction of $2 \mathrm{D}$ convolutional codes.

Let $\mathcal{C}$ be a $1 \mathrm{D}$ convolutional code of rate $k / n$ and different Forney indices $\nu_{1}<\cdots<\nu_{\ell}$ with corresponding multiplicities $m_{1}, \ldots, m_{\ell}$ and

$$
G(z)=\sum_{i=0}^{\nu_{\ell}} G_{i} z^{i}
$$

a column reduced encoder of $\mathcal{C}$ with column degrees in nondecreasing order. Consider a nonzero codeword $v(z)=G(z) u(z)$ with $u(z) \in \mathbb{F}[z]^{k}$. Writing

$$
u(z)=\sum_{i=0}^{\epsilon} u_{i} z^{i} \quad \text { and } \quad v(z)=\sum_{i=0}^{\nu_{\ell}+\epsilon} v_{i} z^{i}
$$

we have

$$
\left\{\begin{aligned}
& v_{0}=G_{0} u_{0}, \\
& v_{1}=G_{0} u_{1}+G_{1} u_{0}, \\
& \vdots \\
& v_{\nu_{\ell}}=G_{0} u_{\nu_{\ell}}+\cdots+G_{\nu_{\ell}} u_{0}, \\
& \vdots \\
& v_{\epsilon} \quad=G_{0} u_{\epsilon}+\cdots+G_{\nu_{\ell}} u_{\epsilon-\nu_{\ell}} \\
& \\
& \quad \vdots \\
& v_{\epsilon+\nu_{\ell}}=G_{\nu_{\ell}} u_{\epsilon}
\end{aligned}\right.
$$


So, we may write

$$
\left[\begin{array}{c}
v_{0} \\
v_{1} \\
v_{2} \\
\vdots \\
v_{\nu_{\ell}+\epsilon}
\end{array}\right]=\mathcal{G}(\epsilon) \quad\left[\begin{array}{c}
u_{\epsilon} \\
\vdots \\
u_{1} \\
u_{0}
\end{array}\right]
$$

where

$$
\mathcal{G}(\epsilon)=\left[\begin{array}{cccccc}
0 & 0 & \cdots & 0 & 0 & G_{0} \\
0 & 0 & \cdots & 0 & G_{0} & G_{1} \\
\vdots & \vdots & \ddots & \vdots & \vdots & \vdots \\
0 & 0 & \cdots & G_{\nu_{\ell}-2} & G_{\nu_{\ell}-1} & G_{\nu_{\ell}} \\
0 & 0 & \cdots & G_{\nu_{\ell}-1} & G_{\nu_{\ell}} & 0 \\
0 & 0 & \cdots & G_{\nu_{\ell}} & 0 & 0 \\
\vdots & \vdots & \ddots & \vdots & \vdots & \vdots \\
G_{0} & G_{1} & \cdots & 0 & 0 & 0 \\
G_{1} & G_{2} & \cdots & 0 & 0 & 0 \\
\vdots & \vdots & \ddots & \vdots & \vdots & \vdots \\
G_{\nu_{\ell}-1} & G_{\nu_{\ell}} & \cdots & 0 & 0 & 0 \\
G_{\nu_{\ell}} & 0 & \cdots & 0 & 0 & 0
\end{array}\right] \in \mathbb{F}^{n\left(\nu_{\ell}+\epsilon+1\right) \times k(\epsilon+1)}
$$

In [2] conditions on $G(z)$, and more specifically on the matrix $\mathcal{G}(\epsilon)$ (for a particular value of $\epsilon)$, were derived in order to obtain an optimal $\left(n, k, \nu_{1}, m_{1}\right)$ convolutional code. We are going to use this result in the main theorem in Section 4 , so we state it here again.

Theorem 5 [2, Theorem 4.1] Let $G(z)=\sum_{i>0} G_{i} z^{i} \in \mathbb{F}[z]^{n \times k}$ be a matrix with distinct column degrees $\nu_{1}<\cdots<\nu_{\ell}$ with multiplicities $m_{1}, \ldots, m_{\ell}$, respectively, and such that all entries of the last $m_{j}+\cdots+m_{\ell}$ columns of $G_{i}$ are nonzero for $i \leq \nu_{j}, j=1, \ldots, \ell$. Suppose that $\mathcal{G}\left(\epsilon_{0}\right)$, defined in (3), is superregular for

$$
\epsilon_{0}=\left\lceil\frac{n\left(\nu_{1}+1\right)-m_{1}}{n-k}\right\rceil-1 .
$$

Then $G(z)$ is column reduced and $\mathcal{C}=\operatorname{Im}_{\mathbb{F}[z]} G(z)$ is an optimal $\left(n, k, \nu_{1}, m_{1}\right)$ convolutional code, i.e.,

$$
\operatorname{dist}(\mathcal{C})=n\left(\nu_{1}+1\right)-m_{1}+1 .
$$

\section{Constructions of MDS 2D convolutional codes}

In this section we present $2 \mathrm{D}$ convolutional codes of rate $k / n$ and degree $\delta$ having the property that all its horizontal projections are optimal 1D convolutional codes. Moreover, summing up the distances of some of these lines we 
show that these codes are in fact MDS $2 \mathrm{D}$ convolutional codes of rate $k / n$ and degree $\delta$, with the restriction that $n>k+\delta$. This restriction is more general than the one presented in [5] for constructing MDS 2D convolutional codes.

Let $\tilde{\nu}=\left\lfloor\frac{\delta}{k}\right\rfloor$. As mentioned in Section 2.2, an MDS 2D convolutional code of rate $k / n$ and degree $\delta$ will have an encoder (with minimal external degree) with $k(\tilde{\nu}+1)-\delta$ columns of degree $\tilde{\nu}$ and $\delta-k \tilde{\nu}$ columns of degree $\tilde{\nu}+1$. Given such an encoder

$$
G\left(z_{1}, z_{2}\right)=\sum_{0 \leq a+b \leq \tilde{\nu}+1} G_{a, b} z_{1}^{a} z_{2}^{b} \in \mathbb{F}\left[z_{1}, z_{2}\right]^{n \times k},
$$

we can write

$$
G\left(z_{1}, z_{2}\right)=\sum_{j=0}^{\tilde{\nu}+1} G_{j}\left(z_{1}\right) z_{2}^{j}
$$

with $G_{j}\left(z_{1}\right)=\sum_{i=0}^{\tilde{\nu}+1} G_{i, j} z_{1}^{i}, i=0,1, \ldots, \tilde{\nu}+1$. Let us consider that the column degrees of $G\left(z_{1}, z_{2}\right)$ are in nondecreasing order. Notice that, if $k \mid \delta$, then $G_{i, j}=0$ for $i+j=\tilde{\nu}+1$. If $k \nmid \delta$ we denote by $\widetilde{G}_{\tilde{\nu}+1}\left(z_{1}\right)$ the submatrix of $G_{\tilde{\nu}+1}\left(z_{1}\right)$ formed by its first $\delta-k \tilde{\nu}$ columns.

Analogously, given $u\left(z_{1}, z_{2}\right) \in \mathbb{F}\left[z_{1}, z_{2}\right]$ and $v\left(z_{1}, z_{2}\right)=G\left(z_{1}, z_{2}\right) u\left(z_{1}, z_{2}\right)$, we can write them in the same way, i.e.,

$$
u\left(z_{1}, z_{2}\right)=\sum_{j=0}^{\ell} u_{j}\left(z_{1}\right) z_{2}^{j} \text { and } v\left(z_{1}, z_{2}\right)=\sum_{j=0}^{\tilde{\nu}+1+\ell} v_{j}\left(z_{1}\right) z_{2}^{j}
$$

for some integer $\ell \geq 0$. Considering $u_{a}\left(z_{1}\right)=0$ if $a>\ell$ or if $a<0$, we obtain

1. If $0 \leq s \leq \tilde{\nu}$,

$$
v_{s}\left(z_{1}\right)=\sum_{j=0}^{s} G_{j}\left(z_{1}\right) u_{s-j}\left(z_{1}\right)
$$

2. If $\tilde{\nu}+1 \leq s \leq \ell$,

$$
v_{s}\left(z_{1}\right)=\sum_{j=0}^{\tilde{\nu}+1} G_{j}\left(z_{1}\right) u_{s-j}\left(z_{1}\right) ;
$$

3. If $\ell+1 \leq s \leq \ell+1+\tilde{\nu}$,

$$
v_{s}\left(z_{1}\right)=\sum_{j=s-\ell}^{\tilde{\nu}+1} G_{j}\left(z_{1}\right) u_{s-j}\left(z_{1}\right) .
$$

Remark 1 Notice that if $\ell<\tilde{\nu}$, both conditions 1 . and 3. above give the same expression for $v_{s}\left(z_{1}\right)$, when $\ell+1 \leq s \leq \tilde{\nu}$. 
If $\ell \neq 0$, for each $s \in\{0,1,2, \ldots, \ell+\tilde{\nu}+1\}$, we may regard $v_{s}\left(z_{1}\right)$ as codewords of a 1D convolutional code $\mathcal{C}_{s}$ with the following characteristics:

1. If $0 \leq s \leq \tilde{\nu}, \mathcal{C}_{s}$ is a $1 \mathrm{D}$ convolutional code of rate $\frac{(s+1) k}{n}$ whose encoder is the matrix

$$
\mathcal{G}_{s}\left(z_{1}\right)=\left[\begin{array}{llll}
G_{0}\left(z_{1}\right) & G_{1}\left(z_{1}\right) & \cdots & G_{s}\left(z_{1}\right)
\end{array}\right] \in \mathbb{F}\left[z_{1}\right]^{n \times(s+1) k},
$$

with column degrees in nondecreasing order. The Forney indices of $\mathcal{C}_{s}$ are $\nu_{i}=\tilde{\nu}+i-1-s$, for $i \in\{1,2, \ldots, s+2\}$ where $\nu_{1}$ has multiplicity $k(\tilde{\nu}+1)-\delta$, $\nu_{i}, i \in\{2, \ldots, s+1\}$, has multiplicity $k$ and $\nu_{s+2}$ has multiplicity $\delta-k \tilde{\nu}$. Notice that if $k \mid \delta$ the Forney indices of $\mathcal{C}_{s}$ would be $\tilde{\nu}, \tilde{\nu}-1, \ldots, \tilde{\nu}-s$ all with multiplicity $k$.

2. If $\tilde{\nu}+1 \leq s \leq \ell$ and $k \nmid \delta$, then $\mathcal{C}_{s}$ is a $1 \mathrm{D}$ convolutional code of rate $\frac{k+\delta}{n}$ whose encoder is the matrix

$$
\mathcal{G}_{s}\left(z_{1}\right)=\left[\begin{array}{lllll}
G_{0}\left(z_{1}\right) & G_{1}\left(z_{1}\right) & \cdots & G_{\tilde{\nu}}\left(z_{1}\right) & \widetilde{G}_{\tilde{\nu}+1}\left(z_{1}\right)
\end{array}\right] \in \mathbb{F}\left[z_{1}\right]^{n \times(k+\delta)},
$$

with column degrees in nondecreasing order. The Forney indices of this code are $\nu_{i}=i-1, i \in\{1,2, \ldots, \tilde{\nu}+2\}$, where $\nu_{i}, i \in\{1, \ldots, \tilde{\nu}+1\}$, has multiplicity $k$ and $\nu_{\tilde{\nu}+2}$ has multiplicity $\delta-k \tilde{\nu}$.

3. If $\tilde{\nu}+1 \leq s \leq \ell$ and $k \mid \delta$, then $\mathcal{C}_{s}$ is a $1 \mathrm{D}$ convolutional code of rate $\frac{k(\tilde{\nu}+1)}{n}$ whose encoder is the matrix

$$
\mathcal{G}_{s}\left(z_{1}\right)=\left[\begin{array}{llll}
G_{0}\left(z_{1}\right) & G_{1}\left(z_{1}\right) & \cdots & G_{\tilde{\nu}}\left(z_{1}\right)
\end{array}\right] \in \mathbb{F}\left[z_{1}\right]^{n \times(k(\tilde{\nu}+1))},
$$

with column degrees in nondecreasing order. The Forney indices of this code are $\nu_{i}=i-1$ for $i \in\{1, \ldots, \tilde{\nu}+1\}$, all with multiplicity $k$.

4. If $\ell+1 \leq s \leq \tilde{\nu}+\ell+1$ and $k \nmid \delta$, then $\mathcal{C}_{s}$ is a $1 \mathrm{D}$ convolutional code of rate $\frac{(\ell-s+1) k+\delta}{n}$ whose encoder is the matrix

$$
\mathcal{G}_{s}\left(z_{1}\right)=\left[\begin{array}{lllll}
G_{s-\ell}\left(z_{1}\right) & G_{s-\ell+1}\left(z_{1}\right) & \cdots & G_{\tilde{\nu}}\left(z_{1}\right) & \widetilde{G}_{\tilde{\nu}+1}\left(z_{1}\right)
\end{array}\right]
$$

with column degrees in nondecreasing order. The Forney indices of this code are $\nu_{i}=i-1$ for $i \in\{1, \ldots, \tilde{\nu}+2+\ell-s\}$, where $\nu_{\tilde{\nu}+2+\ell-s}$, has multiplicity $\delta-k \tilde{\nu}$ and all the others have multiplicity $k$.

5. If $\ell+1 \leq s \leq \tilde{\nu}+\ell$ and $k \mid \delta$, then $\mathcal{C}_{s}$ is a $1 \mathrm{D}$ convolutional code of rate $\frac{(\ell-s+1+\tilde{\nu}) k}{n}$ whose encoder is the matrix

$$
\mathcal{G}_{s}\left(z_{1}\right)=\left[\begin{array}{llll}
G_{s-\ell}\left(z_{1}\right) & G_{s-\ell+1}\left(z_{1}\right) & \cdots & G_{\tilde{\nu}}\left(z_{1}\right)
\end{array}\right]
$$

with column degrees in nondecreasing order. The Forney indices of this code are $\nu_{i}=i-1$ for $i \in\{1, \ldots, \tilde{\nu}+1+\ell-s\}$ all with multiplicity $k$.

If $\ell=0$, let us write instead

$$
\begin{aligned}
u\left(z_{1}, z_{2}\right) & =u_{0}\left(z_{1}\right) \\
& =u_{0,0}+u_{1,0} z_{1}+u_{2,0} z_{1}^{2}+\cdots+u_{\ell_{0}, 0} z_{1}^{\ell_{0}},
\end{aligned}
$$




$$
v\left(z_{1}, z_{2}\right)=\sum_{0 \leq i+j \leq \tilde{\nu}+1+\ell_{0}} v_{i, j} z_{1}^{i} z_{2}^{j}
$$

and

$$
G\left(z_{1}, z_{2}\right)=\sum_{i=0}^{\tilde{\nu}+1} G_{i}^{\prime}\left(z_{2}\right) z_{1}^{i}
$$

with

$$
G_{i}^{\prime}\left(z_{2}\right)=\sum_{j=0}^{\tilde{\nu}+1-i} G_{i, j} z_{2}^{j}, i \in\{0,1, \ldots, \tilde{\nu}+1\}
$$

Consider also

$$
\bar{G}_{0}=\left[\begin{array}{c}
G_{0,0} \\
G_{0,1} \\
\vdots \\
G_{0, \tilde{\nu}} \\
G_{0, \tilde{\nu}+1}
\end{array}\right], \quad \bar{G}_{1}=\left[\begin{array}{c}
G_{1,0} \\
G_{1,1} \\
\vdots \\
G_{1, \tilde{\nu}-1} \\
G_{1, \tilde{\nu}} \\
O_{1}
\end{array}\right], \quad \bar{G}_{i}=\left[\begin{array}{c}
G_{i, 0} \\
G_{i, 1} \\
\vdots \\
G_{i, \tilde{\nu}-i} \\
G_{i, \tilde{\nu}+1-i} \\
O_{1} \\
\vdots \\
O_{i}
\end{array}\right]
$$

for $i \in\{2,3, \ldots, \tilde{\nu}+1\}$, and where each matrix $O_{j}$, is a null $n \times k$ matrix. If $k \nmid \delta$ and $i \in\{0,1, \ldots, \tilde{\nu}+1\}$ the last $k(\tilde{\nu}+1)-\delta$ columns of $G_{i, \tilde{\nu}+1-i}$ are 
also null columns. Then

$$
\left[\begin{array}{c}
v_{0,0} \\
\vdots \\
v_{0, \tilde{\nu}+1} \\
\vdots \\
v_{\ell_{0}, 0} \\
\vdots \\
v_{\ell_{0}, \tilde{\nu}+1} \\
v_{\ell_{0}+1,0} \\
\vdots \\
v_{\ell_{0}+1, \tilde{\nu}} \\
0 \\
v_{\ell_{0}+2,0} \\
\vdots \\
v_{\ell_{0}+2, \tilde{\nu}-1} \\
0 \\
0 \\
v_{\ell_{0}+3,0} \\
\vdots \\
v_{\ell_{0}+\tilde{\nu}+1,0} \\
0 \\
\vdots \\
0
\end{array}\right]=\mathcal{G}\left(n, k, \delta, \ell_{0}\right)\left[\begin{array}{c}
u_{\ell_{0}, 0} \\
\vdots \\
u_{1,0} \\
u_{0,0}
\end{array}\right]
$$

where

$$
\mathcal{G}\left(n, k, \delta, \ell_{0}\right)=\left[\begin{array}{ccccccccccc}
0 & 0 & \cdots & 0 & 0 & \cdots & 0 & 0 & \cdots & 0 & \bar{G}_{0} \\
0 & 0 & \cdots & 0 & 0 & \cdots & 0 & 0 & \cdots & \bar{G}_{0} & \bar{G}_{1} \\
\vdots & \vdots & \ddots & \vdots & \vdots & \ddots & \vdots & \vdots & \ddots & \vdots & \vdots \\
0 & 0 & \cdots & 0 & 0 & \cdots & 0 & \bar{G}_{0} & \cdots & \overline{\bar{G}}_{\tilde{\nu}} & \bar{G}_{\tilde{\nu}+1} \\
0 & 0 & \cdots & 0 & 0 & \cdots & \bar{G}_{0} & \bar{G}_{1} & \cdots & \bar{G}_{\tilde{\nu}+1} & 0 \\
\vdots & \vdots & \ddots & \vdots & \vdots & \ddots & \vdots & \vdots & \ddots & \vdots & \vdots \\
\bar{G}_{0} & \bar{G}_{1} & \cdots & \bar{G}_{\tilde{\nu}} & \bar{G}_{\tilde{\nu}+1} & \cdots & 0 & 0 & \cdots & 0 & 0 \\
\bar{G}_{1} & \bar{G}_{2} & \cdots & \bar{G}_{\tilde{\nu}+1} & 0 & \cdots & 0 & 0 & \cdots & 0 & 0 \\
\vdots & \vdots & \ddots & \vdots & \vdots & \ddots & \vdots & \vdots & \ddots & \vdots & \vdots \\
\bar{G}_{\tilde{\nu}} & \bar{G}_{\tilde{\nu}+1} & \cdots & 0 & 0 & \cdots & 0 & 0 & \cdots & 0 & 0 \\
\bar{G}_{\tilde{\nu}+1} & 0 & \cdots & 0 & 0 & \cdots & 0 & 0 & \cdots & 0 & 0
\end{array}\right] .
$$

Figure 1 illustrates the structure of $\mathcal{G}(n, 4,10,5)$ in which the elements of $\mathcal{G}(n, 4,10,5)$ inside the boxes are different from zero and the other elements are zero. 


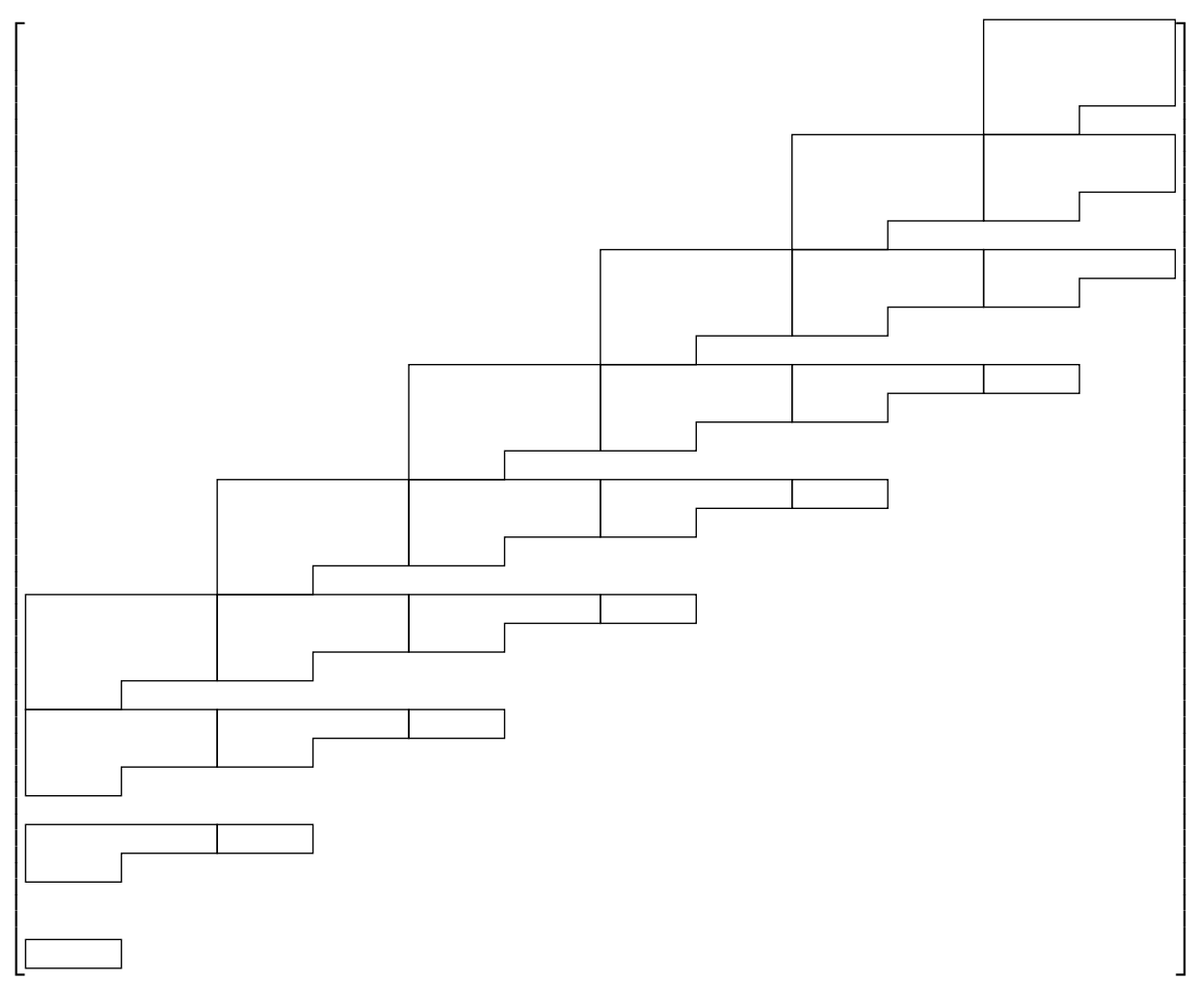

Fig. 1 The structure of $\mathcal{G}(n, 4,10,5)$

Next theorem shows that if the $1 \mathrm{D}$ convolutional codes $\mathcal{C}_{s}$ are optimal, for $s \in\{0,1, \ldots, \tilde{\nu}, \tilde{\nu}+\ell\}$ and the matrix in (13) is superregular, then the 2D convolutional code $\mathcal{C}=\operatorname{Im}_{\mathbb{F}\left[z_{1}, z_{2}\right]} G\left(z_{1}, z_{2}\right)$ is $\operatorname{MDS}$.

Theorem 6 Let $\mathcal{C}$ be a $2 D$ convolutional code of rate $k / n$ and degree $\delta$, such that $n>k+\delta$, and let $G\left(z_{1}, z_{2}\right)=\sum_{j=0}^{\tilde{\nu}+1} G_{j}\left(z_{1}\right) z_{2}^{j}=\sum_{0 \leq i+j \leq \tilde{\nu}+1} G_{i, j} z_{1}^{i} z_{2}^{j} \in$ $\mathbb{F}\left[z_{1}, z_{2}\right]^{n \times k}$ be an encoder of $\mathcal{C}$, that satisfies the following conditions:

1. The first $\delta-k \tilde{\nu}$ columns of $G\left(z_{1}, z_{2}\right)$ have degree $\tilde{\nu}+1$ and the remaining columns have degree $\tilde{\nu}$, where $\tilde{\nu}=\left\lfloor\frac{\delta}{k}\right\rfloor$.

2. For $0 \leq s \leq \tilde{\nu}$ the code $\mathcal{C}_{s}$, defined above, is an optimal $(n,(s+1) k, \tilde{\nu}-$ $s, k(\tilde{\nu}+1)-\delta)$ convolutional code.

3. (a) If $k \nmid \delta$, the $(n, k(\tilde{\nu}+2)-\delta, 0, k)$ convolutional code with encoder $\left[G_{\tilde{\nu}}\left(z_{1}\right) \tilde{G}_{\tilde{\nu}+1}\left(z_{1}\right)\right]$ is optimal, where $\tilde{G}_{\tilde{\nu}+1}\left(z_{1}\right)$ is the submatrix of $G_{\tilde{\nu}+1}\left(z_{1}\right)$ constituted by its first $\delta-k \tilde{\nu}$ columns.

(b) If $k \mid \delta$, the $(n, k, 0, k)$ convolutional code with encoder $G_{\tilde{\nu}}\left(z_{1}\right)$ is optimal. 
4. The matrix $\mathcal{G}\left(n, k, \delta, \ell_{0}\right)$ defined in (13) is superregular, all the entries $G_{i, j}$ are nonzero, for $i \in\{0,1, \ldots, \tilde{\nu}+1\}$ and $j \in\{0,1, \ldots, \tilde{\nu}-i\}$ and all the entries of the first $\delta-k \tilde{\nu}$ columns of $G_{i, \tilde{\nu}+1-i}$ are also nonzero.

Then $\mathcal{C}$ is an MDS $2 D$ convolutional code.

Proof To prove that $\mathcal{C}$ is MDS we have to prove that all nonzero codewords of $\mathcal{C}$ have weight greater or equal than

$$
\frac{(\tilde{\nu}+1)(\tilde{\nu}+2)}{2} n-k(\tilde{\nu}+1)+\delta+1 .
$$

The codewords of the code $\mathcal{C}$ are $v\left(z_{1}, z_{2}\right)=G\left(z_{1}, z_{2}\right) u\left(z_{1}, z_{2}\right)$, where

$$
u\left(z_{1}, z_{2}\right)=\sum_{j=0}^{\ell} u_{j}\left(z_{1}\right) z_{2}^{j}
$$

and

or

$$
v\left(z_{1}, z_{2}\right)=\sum_{j=0}^{\tilde{\nu}+1+\ell} v_{j}\left(z_{1}\right) z_{2}^{j}, \text { if } k \nmid \delta
$$

$$
v\left(z_{1}, z_{2}\right)=\sum_{j=0}^{\tilde{\nu}+\ell} v_{j}\left(z_{1}\right) z_{2}^{j}, \text { if } k \mid \delta .
$$

We can assume without loss of generality that $u_{0}\left(z_{1}\right) \neq 0$ and $u_{\ell}\left(z_{1}\right) \neq 0$.

Suppose first that $\ell \neq 0$. Since we have $u_{0}\left(z_{1}\right) \neq 0$ and $u_{\ell}\left(z_{1}\right) \neq 0$, then $v_{j}\left(z_{1}\right) \in \mathcal{C}_{j}, j=0, \ldots, \tilde{\nu}$, and $v_{\tilde{\nu}+\ell}\left(z_{1}\right) \in \mathcal{C}_{\tilde{\nu}+\ell}$ are nonzero. Therefore,

$$
\operatorname{wt}\left(v\left(z_{1}, z_{2}\right)\right) \geq \operatorname{dist}\left(\mathcal{C}_{\tilde{\nu}+\ell}\right)+\sum_{s=0}^{\tilde{\nu}} \operatorname{dist}\left(\mathcal{C}_{s}\right)
$$

Since, for $0 \leq s \leq \tilde{\nu}, \mathcal{C}_{s}$ is an optimal $(n,(s+1) k, \tilde{\nu}-s, k(\tilde{\nu}+1)-\delta)$ convolutional code, then, by theorem 5

$$
\operatorname{dist}\left(\mathcal{C}_{s}\right)=n(\tilde{\nu}-s+1)-k(\tilde{\nu}+1)+\delta+1 .
$$

If $k \mid \delta, \mathcal{C}_{\tilde{\nu}+\ell}$ has $G_{\tilde{\nu}}\left(z_{1}\right)$ as encoder and therefore it is an optimal $(n, k, 0, k)$ convolutional code and if $k \nmid \delta, \mathcal{C}_{\tilde{\nu}+\ell}$ has $\left[G_{\tilde{\nu}}\left(z_{1}\right) \tilde{G}_{\tilde{\nu}+1}\left(z_{1}\right)\right]$ as encoder and consequently it is an optimal $(n, k+\delta-k \tilde{\nu}, 0, k)$ convolutional code. Thus, in both cases, by theorem $5, \operatorname{dist}\left(\mathcal{C}_{\tilde{\nu}+\ell}\right)=n-k+1$. Therefore, since $n>k(\tilde{\nu}+1)$ and $k(\tilde{\nu}+1)-\delta \leq k$, we obtain

$$
\begin{aligned}
\operatorname{wt}\left(v\left(z_{1}, z_{2}\right)\right) & \geq n-k+1+\sum_{s=0}^{\tilde{\nu}}(n(\tilde{\nu}-s+1)-k(\tilde{\nu}+1)+\delta+1) \\
& \geq k \tilde{\nu}+n \frac{(\tilde{\nu}+1)(\tilde{\nu}+2)}{2}-(\tilde{\nu}+1)(k(\tilde{\nu}+1)-\delta-1) \\
& \geq k \tilde{\nu}+n \frac{(\tilde{\nu}+1)(\tilde{\nu}+2)}{2}-\tilde{\nu}(k(\tilde{\nu}+1)-\delta)-k(\tilde{\nu}+1)+\delta+1 \\
& \geq n \frac{(\tilde{\nu}+1)(\tilde{\nu}+2)}{2}-k(\tilde{\nu}+1)+\delta+1 .
\end{aligned}
$$


Let us now consider $\ell=0$. Then the coefficients of

$$
v\left(z_{1}, z_{2}\right)=\sum_{(i, j) \in \mathbb{N}^{2}} v_{i, j} z_{1}^{i} z_{2}^{j}
$$

satisfy (12). It is easy to check that each column of $\mathcal{G}\left(n, k, \ell_{0}\right)$ has at least

$$
n \frac{(\tilde{\nu}+1)(\tilde{\nu}+2)}{2}
$$

nonzero entries. Suppose that $\operatorname{wt}\left(u_{0}\left(z_{2}\right)\right)=t$. Let $B$ be the matrix formed by the $t$ columns of $\mathcal{G}\left(n, k, \ell_{0}\right)$ that are multiplied by the nonzero entries of $u_{0}\left(z_{1}\right)$ in (12). Then $B$ has at least

$$
n \frac{(\tilde{\nu}+1)(\tilde{\nu}+2)}{2}+\left\lfloor\frac{t+\delta-k \tilde{\nu}}{k}\right\rfloor n
$$

rows with nonzero entries (if $k \mid \delta$ we obtain more nonzero entries). The term $\left\lfloor\frac{t+\delta-k \tilde{\nu}}{k}\right\rfloor n$ accounts for the fact that if the number of columns increases, this does not always mean that the number of rows, with nonzero entries, also increases. However, if the number of columns is increased by $k$, the number of rows with nonzero entries must increase by at least $n$ (in fact, in most of the cases, the number of rows increases by more than $n$ ).

Since $\mathcal{G}\left(n, k, \ell_{0}\right)$ is superregular, by theorem 2 , it follows that

$$
\operatorname{wt}\left(v\left(z_{1}, z_{2}\right)\right) \geq n \frac{(\tilde{\nu}+1)(\tilde{\nu}+2)}{2}-k(\tilde{\nu}+1)+\delta+1 .
$$

Hence,

$$
\operatorname{dist}(\mathcal{C}) \geq n \frac{(\tilde{\nu}+1)(\tilde{\nu}+2)}{2}-k(\tilde{\nu}+1)+\delta+1,
$$

and $\mathcal{C}$ is an MDS 2D convolutional code.

Next, we construct a $2 \mathrm{D}$ convolutional code that is MDS of rate $k / n$ and degree $\delta$ with $n>k+\delta$, by constructing an encoder $G\left(z_{1}, z_{2}\right) \in \mathbb{F}\left[z_{1}, z_{2}\right]^{n \times k}$ that satisfies the conditions of theorem 6 .

The encoder of $\mathcal{C}_{\tilde{\nu}+1}$ is the matrix

$$
\begin{aligned}
& \mathfrak{G}=\left[\begin{array}{lllll}
G_{0}\left(z_{1}\right) & G_{1}\left(z_{1}\right) & \cdots & G_{\tilde{\nu}}\left(z_{1}\right) & \widetilde{G}_{\tilde{\nu}+1}\left(z_{1}\right)
\end{array}\right] \\
& =\widehat{G}_{0}+\widehat{G}_{1} z_{1}+\cdots+\widehat{G}_{\tilde{\nu}+1} z_{1}^{\tilde{\nu}+1},
\end{aligned}
$$

where, for $0 \leq i \leq \tilde{\nu}+1$, the $n \times(k+\delta)$ matrix $\widehat{G}_{i}$ is defined as

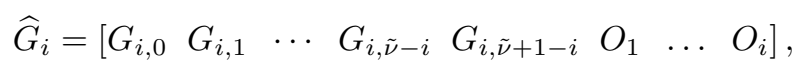

where, $O_{j}$, is a null $n \times k$ matrix, for $j<i, O_{i}$ is a null $n \times(\delta-k \tilde{\nu})$ matrix and the last $k(\tilde{\nu}+1)-\delta$ columns of $G_{i, \tilde{\nu}+1-i}$ are also null columns.

If the following matrix $\mathcal{G}(\epsilon)$ 


$$
\mathcal{G}(\epsilon)=\left[\begin{array}{cccccc}
0 & 0 & \cdots & 0 & 0 & \widehat{G}_{0} \\
0 & 0 & \cdots & 0 & \widehat{G}_{0} & \widehat{G}_{1} \\
\vdots & \vdots & \ddots & \vdots & \vdots & \vdots \\
0 & 0 & \cdots & \widehat{G}_{\tilde{\nu}-1} & \widehat{G}_{\tilde{\nu}} & \widehat{G}_{\tilde{\nu}+1} \\
0 & 0 & \cdots & \widehat{G}_{\tilde{\nu}} & \widehat{G}_{\tilde{\nu}+1} & 0 \\
0 & 0 & \cdots & \widehat{G}_{\tilde{\nu}+1} & 0 & 0 \\
\vdots & \vdots & \ddots & \vdots & \vdots & \vdots \\
\widehat{G}_{0} & \widehat{G}_{1} & \cdots & 0 & 0 & 0 \\
\widehat{G}_{1} & \widehat{G}_{2} & \cdots & 0 & 0 & 0 \\
\vdots & \vdots & \ddots & \vdots & \vdots & \vdots \\
\widehat{G}_{\tilde{\nu}} & \widehat{G}_{\tilde{\nu}+1} & \cdots & 0 & 0 & 0 \\
\widehat{G}_{\tilde{\nu}+1} & 0 & \cdots & 0 & 0 & 0
\end{array}\right] \in \mathbb{F}^{n(\tilde{\nu}+\epsilon+2) \times(k+\delta)(\epsilon+1)}
$$

where

$$
\epsilon=\left\lceil\frac{n(\tilde{\nu}+1)-k(\tilde{\nu}+1)+\delta}{n-(k+\delta)}\right\rceil-1,
$$

is superregular then, for any $\ell \in \mathbb{N}$, the corresponding matrices of the encoders $\mathcal{G}_{s}$ of the convolutional codes $\mathcal{C}_{s}$, for $s \in\{0,1, \ldots, \tilde{\nu}+\ell+1\}$ are also superregular, since they are submatrices of $\mathcal{G}(\epsilon)$. Therefore, by theorem 5 , all the $1 \mathrm{D}$ convolutional codes $\mathcal{C}_{s}$, with $s \in\{0,1, \ldots, \tilde{\nu}+\ell+1\}$ are optimal convolutional codes.

For example, if $0 \leq s \leq \tilde{\nu}$, the encoder of $\mathcal{C}_{s}$ is

$$
\begin{aligned}
\mathcal{G}_{s}\left(z_{1}\right) & =\left[\begin{array}{llll}
G_{0}\left(z_{1}\right) & G_{1}\left(z_{1}\right) & \cdots & G_{s}\left(z_{1}\right)
\end{array}\right] \\
& =G_{0}^{(s)}+G_{1}^{(s)} z_{1}+\cdots+G_{\tilde{\nu}+1}^{(s)} z_{1}^{\tilde{\nu}+1},
\end{aligned}
$$

where, for $0 \leq i \leq \tilde{\nu}+1$

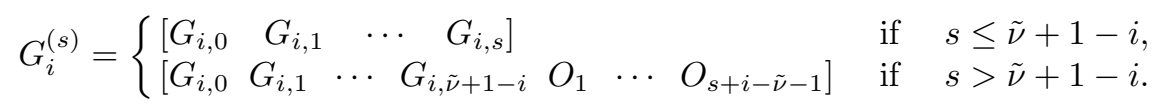

Suppose that $\mathcal{G}(\epsilon)$ is superregular for

$$
\epsilon=\left\lceil\frac{n(\tilde{\nu}+1)-k(\tilde{\nu}+1)+\delta}{n-(k+\delta)}\right\rceil-1,
$$




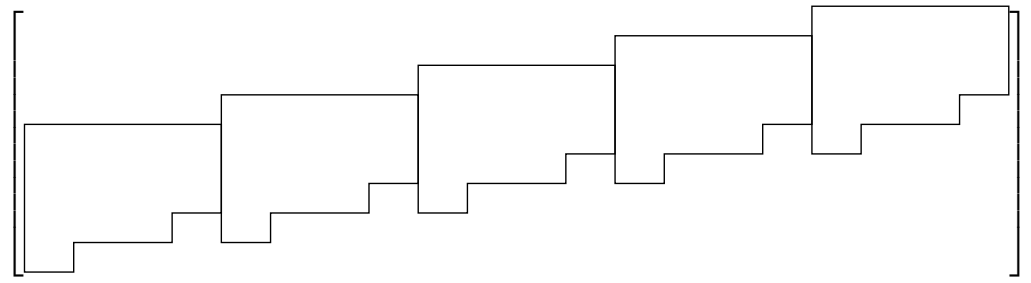

Fig. 2 The structure of $\mathcal{G}(4,1)$

The matrix

$$
\mathcal{G}\left(\epsilon_{0}, s\right)=\left[\begin{array}{cccccc}
0 & 0 & \cdots & 0 & 0 & G_{0}^{(s)} \\
0 & 0 & \cdots & 0 & G_{0}^{(s)} & G_{1}^{(s)} \\
\vdots & \vdots & \ddots & \vdots & \vdots & \vdots \\
0 & 0 & \cdots & G_{\tilde{\nu}-1}^{(s)} & G_{\tilde{\nu}}^{(s)} & G_{\tilde{\nu}+1}^{(s)} \\
0 & 0 & \cdots & G_{\tilde{\nu}}^{(s)} & G_{\tilde{\nu}+1}^{(s)} & 0 \\
0 & 0 & \cdots & G_{\tilde{\nu}+1}^{(s)} & 0 & 0 \\
\vdots & \vdots & \ddots & \vdots & \vdots & \vdots \\
G_{0}^{(s)} & G_{1}^{(s)} & \cdots & 0 & 0 & 0 \\
G_{1}^{(s)} & G_{2}^{(s)} & \cdots & 0 & 0 & 0 \\
\vdots & \vdots & \ddots & \vdots & \vdots & \vdots \\
G_{\tilde{\nu}}^{(s)} & G_{\tilde{\nu}+1}^{(s)} & \cdots & 0 & 0 & 0 \\
G_{\tilde{\nu}+1}^{(s)} & 0 & \cdots & 0 & 0 & 0
\end{array}\right]
$$

where

$$
\epsilon_{0}=\left\lceil\frac{n(\tilde{\nu}-s+1)-k(\tilde{\nu}+1)+\delta}{n-(s+1) k}\right\rceil-1,
$$

is a submatrix of $\mathcal{G}(\epsilon)$, since $(s+1) k \leq \delta+k$ and $G_{i}^{(s)}$ is a submatrix of $\widehat{G}_{i}$. Therefore, $\mathcal{G}\left(\epsilon_{0}, s\right)$ is superregular. Then, by theorem $5, \mathcal{C}_{s}$ is an optimal $(n,(s+1) k, \tilde{\nu}-s, k(\tilde{\nu}+1)-\delta)$ convolutional code.

Figure 2. shows the structure of $\mathcal{G}(4,1)$, where the elements of $\mathcal{G}(4,1)$ inside the boxes are different from zero and the remaining elements are zero.

Similarly, since the encoder of $\mathcal{C}_{\tilde{\nu}+\ell}$, when $k \nmid \delta$ is

$$
\begin{aligned}
\mathcal{G}_{\tilde{\nu}+\ell}\left(z_{1}\right) & =\left[\begin{array}{ll}
G_{\tilde{\nu}}\left(z_{1}\right) & \widetilde{G}_{\tilde{\nu}+1}\left(z_{1}\right)
\end{array}\right] \\
& =G_{0}^{(\tilde{\nu}+\ell)}+G_{1}^{(\tilde{\nu}+\ell)} z_{1},
\end{aligned}
$$

where, if we represent by $\widetilde{G}_{0, \tilde{\nu}+1}$ the matrix formed by the first $\delta-k \tilde{\nu}$ columns of $G_{0, \tilde{\nu}+1}$,

$$
G_{0}^{(\tilde{\nu}+\ell)}=\left[\begin{array}{ll}
G_{0, \tilde{\nu}} & \widetilde{G}_{0, \tilde{\nu}+1}
\end{array}\right]
$$


and

$$
G_{1}^{(\tilde{\nu}+\ell)}=\left[\begin{array}{ll}
G_{1, \tilde{\nu}} & 0
\end{array}\right] .
$$

If the following matrix $\mathcal{G}(\epsilon, \tilde{\nu}+\ell)$

$$
\mathcal{G}(\epsilon, \tilde{\nu}+\ell)=\left[\begin{array}{cccccc}
0 & 0 & \cdots & 0 & 0 & G_{0}^{(\tilde{\nu}+\ell)} \\
0 & 0 & \cdots & 0 & G_{0}^{(\tilde{\nu}+\ell)} & G_{1}^{(\tilde{\nu}+\ell)} \\
0 & 0 & \cdots & G_{0}^{(\tilde{\nu}+\ell)} & G_{1}^{(\tilde{\nu}+\ell)} & 0 \\
\vdots & \vdots & \ddots & \vdots & \vdots & \vdots \\
G_{0}^{(s)} & G_{1}^{(s)} & \cdots & 0 & 0 & 0 \\
G_{1}^{(s)} & 0 & \cdots & 0 & 0 & 0
\end{array}\right]
$$

is superregular then, by theorem $5, \mathcal{C}_{\tilde{\nu}+\ell}$ is an optimal $(n, \delta-k(\tilde{\nu}-1), 0, k)$ convolutional code.

Let $\alpha$ be a primitive element of a finite field $\mathbb{F}$ and for $0 \leq a, b \leq \tilde{\nu}+1$, define $G_{a, b}=\left[g_{i, j}^{(a, b)}\right] \in \mathbb{F}^{n \times k}$ by

$$
g_{i, j}^{(a, b)}=\left\{\begin{array}{cc}
\alpha^{2^{(a(\tilde{\nu}+2)+b) n+i+j-2}} & \text { if } 0 \leq a+b \leq \tilde{\nu}, \\
\alpha^{2^{(a(\tilde{\nu}+2)+b) n+i+j-2}} & \text { if } a+b=\tilde{\nu}+1 \text { and } j \leq \delta-k \tilde{\nu}, \\
0 & \text { if } a+b=\tilde{\nu}+1 \text { and } j>\delta-k \tilde{\nu}, \\
0 & \text { if } \quad a+b>\tilde{\nu}+1 .
\end{array}\right.
$$

With this construction we are now in conditions to state and prove the existence of a $2 \mathrm{D}$ convolutional code $\mathcal{C}$ of rate $k / n$ and degree $\delta$ for $n>k+\delta$, such that its horizontal projections are optimal $1 \mathrm{D}$ convolutional codes and $\mathcal{C}$ is an MDS 2D convolutional code.

Theorem 7 Let $\alpha$ be a primitive element of a finite field $\mathbb{F}=\mathbb{F}_{p^{N}}$. Let $n, k, \delta, \tilde{\nu} \in \mathbb{N}$ such that $k \nmid \delta, \tilde{\nu}=\left\lfloor\frac{\delta}{k}\right\rfloor$ and $n>k+\delta$. Consider $G\left(z_{1}, z_{2}\right)$ as defined in (5) with $G_{a, b}$ defined in (17). Then, for $N \in \mathbb{N}$ sufficiently large, $\mathcal{C}=\operatorname{Im}_{\mathbb{F}\left[z_{1}, z_{2}\right]} G\left(z_{1}, z_{2}\right)$ is a $2 D$ convolutional code of rate $k / n$ and degree $\delta$ such that all its horizontal projections are optimal $1 D$ convolutional codes with the appropriate parameters. In particular, if $\mathcal{C}_{s}$ is the $s$-th horizontal projection, for $0 \leq s \leq \tilde{\nu}$, then $\mathcal{C}_{s}$ is an optimal $1 D(n,(s+1) k, \tilde{\nu}-s, k(\tilde{\nu}+1)-\delta)$ convolutional code.

Proof The matrix $\mathcal{G}(\epsilon)$ defined in (14) satisfies the conditions of the theorem 3 , and so it is superregular. Therefore, all the corresponding matrices of the encoders $\mathcal{G}_{s}$ of the convolutional codes $\mathcal{C}_{s}$, for $s \in\{0,1, \ldots, \tilde{\nu}+\ell+1\}$ are also superregular, for any $\ell \in \mathbb{N}$, since they are submatrices of $\mathcal{G}(\epsilon)$. Hence, by theorem 5 , all the $1 \mathrm{D}$ convolutional codes $\mathcal{C}_{s}$, with $s \in\{0,1, \ldots, \tilde{\nu}+\ell+1\}$ are optimal convolutional codes.

Remark 2 Obviously, switching $z_{1}$ and $z_{2}$ one can construct 2D convolutional codes such that all their vertical projections are optimal 1D convolutional codes. 
The 2D convolutional codes presented here through equation (17) are good candidates when the decoding along (horizontal) lines is relevant. Moreover they have the maximum possible distance among all $2 \mathrm{D}$ convolutional codes with the same rate and degree, as it is shown in the following result.

Corollary 1 Let $\alpha$ be a primitive element of a finite field $\mathbb{F}=\mathbb{F}_{p^{N}}$. Let $n, k, \delta, \tilde{\nu} \in \mathbb{N}$ such that $k \nmid \delta, \tilde{\nu}=\left\lfloor\frac{\delta}{k}\right\rfloor$ and $n>k+\delta$. Consider $G\left(z_{1}, z_{2}\right)$ as defined in (5) with $G_{a, b}$ defined in (17). Then, for $N \in \mathbb{N}$ sufficiently large, $\mathcal{C}=\operatorname{Im}_{\mathbb{F}\left[z_{1}, z_{2}\right]} G\left(z_{1}, z_{2}\right)$ is an MDS $2 D$ convolutional code of rate $k / n$ and degree $\delta$.

Proof Clearly, the matrices $\mathcal{G}\left(n, k, \delta, \ell_{0}\right)$ in $(13), \mathcal{G}(\epsilon, s)$ in $(15)$ for $0 \leq s \leq \tilde{\nu}$, and $\mathcal{G}(\epsilon, \tilde{\nu}+\ell)$ in (16) satisfy the conditions of at least one of the theorems 3 and 4 , and so they are superregular. Then, by theorem 5, conditions 2., 3 . and 4 . of theorem 6 are satisfied. It is immediate to see that condition 1 . of theorem 6 is also fulfilled, and so $\mathcal{C}$ is an MDS $2 \mathrm{D}$ convolutional code of rate $k / n$ and degree $\delta$.

Constructions of MDS 2D convolutional codes of rate $k / n$ and degree $\delta$, when $k \mid \delta$, can be obtained in a similar way by considering minor adjustments.

Acknowledgements The authors are grateful to the anonymous referees for the many insightful comments.

\section{References}

1. P. Almeida, D. Napp, and R. Pinto. A new class of superregular matrices and MDP convolutional codes. Linear Algebra and its Applications, 439(7):2145-2157, 2013.

2. P. Almeida, D. Napp, and R. Pinto. Superregular matrices and applications to convolutional codes. Linear Algebra and its Applications, 499:1-25, 2016.

3. C. Charoenlarpnopparut and N.K. Bose. Grobner bases for problem solving in multidimensional systems. Multidimensional Systems and Signal Processing, 12(3):365-376, 2001.

4. J.J. Climent, D. Napp, C. Perea, and R. Pinto. A construction of MDS 2D convolutional codes of rate $1 / n$ based on superregular matrices. Linear Algebra and its Applications, 437:766-780, 2012

5. J.J. Climent, D. Napp, C. Perea, and R. Pinto. Maximum distance separable 2D convolutional codes. IEEE Trans. Information Theory, 62(2):669-680, 2016.

6. J.J. Climent, D. Napp, R. Pinto, and R. Simões. Decoding of 2D convolutional codes over the erasure channel. Advances in Mathematics of Communications, 10(1):179-193, 2016.

7. E. Fornasini and M.E. Valcher. Algebraic aspects of two-dimensional convolutional codes. IEEE Trans. Inf. Th, 40(4):1068-1082, 1994.

8. H. Gluesing-Luerssen, J. Rosenthal, and R. Smarandache. Strongly MDS convolutional codes. IEEE Trans. Inform. Theory, 52(2):584-598, 2006.

9. J. Hansen, J. Østergaard, J. Kudahl, and J. Madsen. On the construction of jointly superregular lower triangular Toeplitz matrices. International Symposium on Information Theory (ISIT), 2016.

10. R. Hutchinson. The existence of strongly MDS convolutional codes. SIAM Journal on Control and Optimization, 47(6):2812-2826, 2008. 
11. R. Hutchinson, R. Smarandache, and J. Trumpf. On superregular matrices and MDP convolutional codes. Linear Algebra and its Applications, 428:2585-2596, 2008.

12. J. Justesen and S. Forchhammer. Two dimensional information theory and coding. With applications to graphics data and high-density storage media. Cambridge: Cambridge University Press. ix, 171 p., 2010.

13. G. La Guardia. On classical and quantum MDS-convolutional BCH codes. IEEE Trans. Inf. Th., 60(1):304-312, 2013.

14. R.G. Lobo, D. L. Bitzer, and M. A. Vouk. On locally invertible encoders and muldimensional convolutional codes. IEEE Trans. Inf. Th., 58(3):1774-1782, 2012.

15. R. Mahmood, A. Badr, and A. Khisti. Convolutional codes with maximum column sum rank for network streaming. IEEE Trans. Inform. Theory, 62(6):3039-3052, 2016.

16. R. J. McEliece. The algebraic theory of convolutional codes. In V. Pless and W.C. Huffman, editors, Handbook of Coding Theory, volume 1, pages 1065-1138. Elsevier Science Publishers, Amsterdam, The Netherlands, 1998.

17. D. Napp, C. Perea, and R. Pinto. Input-state-output representations and constructions of finite support 2D convolutional codes. Advances in Mathematics of Communications, 4(4):533-545, 2010.

18. D. Napp, R. Pinto, and T. Toste. On MDS convolutional codes over $\mathbb{Z}_{p^{r}}$. Designs, Codes and Cryptography, 83:101-114, 2017.

19. G. Norton. On minimal realization over a finite chain ring. Designs, Codes and Cryptography, 16(2):161-178, 1999

20. M. El Oued and P. Sole. MDS convolutional codes over a finite ring. IEEE Trans. Inf. Th., 59(11):7305 - 7313, 2013

21. Ferruh Ozbudak and Buket Ozkaya. A minimum distance bound for quasi-nd-cyclic codes. Finite Fields and Their Applications, 41:193-222, 2016.

22. T. Pinho, R. Pinto, and P. Rocha. Realization of $2 \mathrm{D}$ convolutional codes of rate $\frac{1}{n}$ by separable Roesser models. Designs, Codes and Cryptography, 70(1):241-250, 2014.

23. J. Rosenthal and E.V. York. BCH convolutional codes. IEEE Trans. Inf. Th., 45(6):1833-1844, 1999.

24. R. M. Roth and A. Lempel. On MDS codes via Cauchy matrices. IEEE Trans. Inf. Th, 35(6):1314-1319, 1989 .

25. R. Smarandache, H. Gluesing-Luerssen, and J. Rosenthal. Constructions of MDSconvolutional codes. IEEE Trans. Automat. Control, 47(5):2045-2049, 2001.

26. V. Tomás. Complete-MDP Convolutional Codes over the Erasure Channel. $\mathrm{PhD}$ thesis, Departamento de Ciencia de la Computación e Inteligencia Artificial, Universidad de Alicante, Alicante, España, July 2010.

27. V. Tomás, J. Rosenthal, and R. Smarandache. Decoding of MDP convolutional codes over the erasure channel. In Proceedings of the 2009 IEEE International Symposium on Information Theory (ISIT 2009), pages 556-560, Seoul, Korea, June 2009. IEEE.

28. V. Tomas, J. Rosenthal, and R. Smarandache. Decoding of convolutional codes over the erasure channel. 58(1):90-108, January 2012.

29. M.E. Valcher and E. Fornasini. On 2D finite support convolutional codes: an algebraic approach. Multidimensional Systems and Signal Processing, 5:231-243, 1994.

30. P. Weiner. Muldimensional Convolutional Codes. PhD dissertation, University of Notre Dame, USA, 1998. 